\title{
Pressure ulcer knowledge, beliefs and practices in a group of South Africans with spinal cord injury
}

\author{
Adri Marica Visser ${ }^{1} \cdot$ Surona Visagie ${ }^{2}$
}

Received: 11 April 2019 / Revised: 2 September 2019 / Accepted: 8 September 2019

(c) The Author(s), under exclusive licence to International Spinal Cord Society 2019

\begin{abstract}
Study design A quantitative, descriptive study using a cross-sectional survey.

Objectives To describe the pressure ulcer knowledge, beliefs and practices amongst persons with SCI, who received rehabilitation at a Cape Town rehabilitation centre.

Setting A rehabilitation centre for clients with physical disabilities in Cape Town, South Africa.

Methods A quantitative, descriptive study, that employed consecutive sampling, was done. Participants included inpatients $(n=30)$, outpatients $(n=33)$ and peer supporters $(n=8)$. Data were collected during April and March 2015 with a questionnaire developed through collating existing questionnaires and adapting it for the study context. This rendered a knowledge score and data on beliefs and practices. The Fisher's exact test was used for comparative analysis $(p<0.05)$.

Results The mean combined knowledge score was $42.7 \%$. The majority of participants (88.7\%) believed pressure ulcers to be serious and $45 \%$ thought they were likely to develop a PU. They believed daily skin checks $(80.3 \%$ ), weight shifting (86\%) and limiting sitting time (80.3\%) could prevent PU development. Study participants indicated that they did not regularly follow guideline recommended practices like regular pressure relief (51\%) (36 participants) or daily skin inspection (38\%) (27 participants) and 37\% (26 participants) reported being current smokers.

Conclusion Participants showed a lack of knowledge, which might have affected their pressure ulcer prevention practices negatively. The study findings can be used to assist with the development of a contextually relevant training programme on pressure care.
\end{abstract}

\section{Introduction}

Pressure ulcers (PUs) are a frequent and serious secondary complication of spinal cord injuries (SCI) [1-4]. They interfere with a person's function and quality of life, and can lead to death [4]. Management of PUs increases the care and financial burden on personal and health systems levels [5, 6].

On a cellular level a PU is caused by pressure, shear or friction forces applied to the skin leading to decreased circulation and soft-tissue necrosis [7, 8]. Infections, anaemia, malnutrition, paralysis, decreased sensation, contractures,

Adri Marica Visser

adri.visser@westerncape.gov.za

1 Western Cape Department of Health, Western Cape Rehabilitation Centre, 103 Highlands drive, Mitchells Plain, Cape Town 7785, South Africa

2 Centre for Rehabilitation Studies, Stellenbosch University, PO Box 40, Fraserburg 6969, South Africa spasticity and incontinence can increase the risk for developing PUs [9].

However, PUs could potentially be prevented through adhering to various skin care practices and ensuring good general health [10-12]. Best practice guidelines on the prevention and management of PU in people with SCIs agree that PU prevention is dependent on knowledge of the aetiology of PUs, caring for and cleaning the skin, incontinence management, skin inspection, position changes, pressure relief practices, appropriate wheelchair and cushion selection and use, nutritional choices, no substance abuse and seeking timely care [10-13].

Adherence to prevention practices is influenced by a person's knowledge, attitudes and beliefs [14]. Knowledge influences health beliefs [15]. Health beliefs in turn, influence the actions one takes to prevent unwanted occurrences, such as developing a PU [15]. Health beliefs are influenced by internal and external cues. Internal cues come from within the body such as symptoms, for instance increased spasticity. External cues come from outside the body and include personal experiences, interaction with others and acquired knowledge [15]. 
Guihan and Bombardier [16] studied PU prevention knowledge and beliefs amongst a group of American veterans. In the study they tested the knowledge of the participants by means of a questionnaire, which then rendered a percentage score of questions answered correctly. Their mean knowledge score was $73.4 \%$.

The current study was performed at a specialised rehabilitation centre in the City of Cape Town, South Africa. No prevalence statistics are available for SCI in South Africa. Joseph and Wikmar [2] studied the secondary complications of clients with traumatic SCI at one of the tertiary hospitals in Cape Town and found PUs to be the most common complication post SCI. They indicated a prevalence rate of $28.9 \%$.

\section{Study setting}

The study setting, a specialised rehabilitation centre for persons with physical disabilities provides 2-3 months of inpatient rehabilitation and outpatient follow up by an interdisciplinary team, for persons who suffered SCI (traumatic and non-traumatic). The rehabilitation programme focuses on the retraining of activities of daily living, mobility, community reintegration, health management and prevention of secondary complications including PUs. Wheelchairs and cushions are routinely provided. Outpatients access the service for replacement cushions and repairs as needed. In the more rural settings of South Africa however, this is not always the case and most persons with SCI who are dependent on the state healthcare service cannot afford to buy their own mobility devices. Those who access private healthcare services usually have limited healthcare insurance and procure their own devices as needed.

This study is unique since it was performed in an upper middle income country. Research of this nature has been conducted in the United States and in Europe and significant progress has been made in how PUs are prevented in those settings in terms of knowledge, beliefs and practices [8-11, 15, 16]. In South Africa, however, we still lack baseline information as the setting is very different in terms of resources, cultural setting, education levels and income.

This study aimed to determine knowledge, beliefs and practices on PUs of persons with SCI that received care at this rehabilitation centre.

\section{Methods}

A cross-sectional survey was done. The study population consisted of persons living with SCI (traumatic or non-traumatic) who received rehabilitation at the centre, or visited an outpatient clinic at the centre between 31 March 2015 and 19 May 2015. In addition, persons who provided peer counselling at the centre in $2015(n=8)$ were included. All persons, older than 18 , who used a manual wheelchair as a primary means of mobility, were included in the study. Persons with cognitive impairments that could limit the ability to remember and/or implement PU education and those who could not self-propel or used motorised wheelchairs were excluded, because past research has found that persons with SCI that self-propel their wheelchairs are at greater risk for PUs than their peers $[17,18]$.

All inpatients that completed rehabilitation during the study period and those with SCI who visited outpatient clinics (and had been discharged for at least 3 months) were consecutively sampled. In total, 71 people participated in the study. See Table 1 for details.

A questionnaire was developed from assessment tools that had been used in a series of studies on PUs by a group of researchers, led by Dr Marylou Guihan from the Spinal Cord Injury Quality Enhancement Research Initiative at the Edward Hines Jr VA Hospital in Hines, IL, United States of America [19]. Permission was obtained to use and adapt the tools to fit the current context.

The revised questionnaire had the following five sections.

(1) Demographic and basic clinical information.

(2) Knowledge of PUs and PU prevention. (The knowledge section consisted of 20 structured questions with the maximum score being 56. In presenting the results score out of 56 is presented as well as percentages, i.e. the score out of 56 stated as a percentage.)

(3) Beliefs about PU and PU prevention. (The beliefs section consisted of 13 questions with forced-choice Likert-type answer scales.)

(4) PU prevention practices. (The practices section consisted of ten questions using Likert-type answer scales. To enable statistical analysis, answers were dichotomised and presented as either doing it as recommended or not.)

(5) Transfer, and pressure relief skills test.
Table 1 Details of participants included in the study

\begin{tabular}{lllll}
\hline & Total & Inpatients & Outpatients & Peer supporters \\
\hline $\begin{array}{l}\text { Population }(N) \\
\text { Excluded due to being inappropriate for the study }\end{array}$ & 93 & 42 & 43 & 8 \\
purposes & $7(7.5 \%)$ & $7(16.7 \%)$ & 0 & 0 \\
$\begin{array}{l}\text { Refused consent } \\
\text { Participants }(n)\end{array}$ & $15(16.1 \%)$ & $5(11.9 \%)$ & $10(23.3 \%)$ & 0 \\
\hline
\end{tabular}


Table 2 Demographic information of participants in percentages (numbers in brackets are actual number of participants)

\begin{tabular}{lllll}
\hline & Total (71) & Inpatients (30) & Outpatients (33) & Peer supporters (8) \\
\hline Mean age & $36.3($ SD 12.3) & $32($ SD 11) & 40.5 (SD 13) & 34.8 (SD 8.4) \\
Range of age & $18-70$ & $18-56$ & $21-70$ & $24-49$ \\
Males & $86 \%(61)$ & $76.7 \%(23)$ & $90.9 \%(30)$ & $100 \%(8)$ \\
Females & $14 \%(10)$ & $23.3 \%(7)$ & $9.1 \%(3)$ & $0 \%(0)$ \\
Formal education & $14 \%(10)$ & $17 \%(5)$ & $15 \%(5)$ & $0 \%(0)$ \\
$\quad \begin{array}{l}\text { No or primary education } \\
\text { Some secondary education }\end{array}$ & $55 \%(39)$ & $57 \%(17)$ & $55 \%(18)$ & $50 \%(4)$ \\
$\quad \begin{array}{l}\text { Completed secondary } \\
\text { education }\end{array}$ & $23 \%(16)$ & $13 \%(4)$ & $30 \%(10)$ & $25 \%(2)$ \\
$\quad \begin{array}{l}\text { Tertiary education } \\
\text { Employment status }\end{array}$ & $8 \%(6)$ & $13 \%(4)$ & $0 \%(0)$ & $25 \%(2)$ \\
$\quad$ Unemployed & $77 \%(55)$ & $80 \%(24)$ & $78.8 \%(26)$ & $62.5 \%(5)$ \\
\hline
\end{tabular}

Content, face and structural validity [20] were ensured with the assistance of five experts and further tested during a pilot study. The final questionnaire was translated and reverse-translated to ensure accuracy of Afrikaans and Xhosa versions. Data were collected by a trained research assistant through face-to-face interviews.

Data were analysed with STATA 14. Descriptive and comparative analysis were done [21]. Due to small values in some cells, data were dichotomized by collapsing of categories (for example terms like very serious and life threatening were combined as were none and a little) and the Fischer's exact test was used. Alpha 0.05 was considered statistically significant.

\section{Limitations}

(1) Caution is needed when generalising results to other clients at the Centre and other urban public funded rehabilitation settings in South Africa since sampling was not systematic.

(2) The instrument used for data collection was not tested for reliability and criterion validity.

\section{Results}

\section{Demographic and medical information}

Table 2 summarises the participants demographic profile. The mean age was 36.3 (standard deviation 12.3). The outpatients (40.5; standard deviation 13) were slightly older than inpatients (32; standard deviation 11) and peer supporters (34.8; standard deviation 8.4). The majority of participants had some high school education (55\%) and were unemployed $(77 \%)$.

Time since injury varied between participants with a range of less than 6 months (mostly in patients) to more than 5 years (mostly peer supporters). The majority of participants $(89 \%)$ suffered traumatic injuries below the level of T1 $(76 \%)$.

According to Fig. 1, 57\% of participants never had a PU, while $4 \%$ had continuous problems with PUs. The inpatients who participated in the study had not been discharged home since their injury, thus these nine participants developed their ulcers in hospital. Of the outpatients, eight reported that their PUs had developed before discharge home and ten reported the PUs developed post discharge (Table 3).

\section{Knowledge}

As shown in Table 4, the combined mean knowledge score was 23.9 out of a possible $56(42.7 \%$ ) (standard deviation 7.8). Peer supporters (65.2\%; standard deviation 36.5) had the highest mean score followed by inpatients $(40.4 \%$; standard deviation 22.6) and outpatients (39.4\%; standard deviation 22.1).

The aspects that created the greatest knowledge challenges were knowledge about nutrition (none of the participants scored $100 \%$ ), the risk factors of PU (one participant scored $100 \%$ ), the time it takes for a PU to develop (four participants scored $100 \%$ ), general prevention practices (seven participants scored 100\%) and the description of stages of a PU (eight participants answered it correctly). Less than $20 \%$ of inpatients and outpatients mentioned skin checks, a balanced diet, the use of a cushion, not wearing overly tight clothes, maintaining good overall health, using lotion on dry skin and sleeping on a special mattress as practices that can prevent PUs. Forty-three percent (43\%) of 
inpatients, $67 \%$ of outpatients and $100 \%$ of peer supporters knew that sitting too long increased the risk for PUs. Less than $25 \%$ of all participants identified shear as a PU cause. Knowledge about the recurring nature of PUs scored the highest (61 of the participants answered correctly) followed by knowledge on the type of cushion ( 55 of the participants answered correctly), the actions that should be taken when noticing the first sign of a PU (51 participants answered correctly) and the question of whether weight shifts should still be performed on a speciality cushion (49 participants answered correctly). Peer supporters achieved the highest scores in all the knowledge categories, but due to their small number this did not significantly impact the mean knowledge score. Excluding them, results in a $2.8 \%$ lower mean score.

\section{Beliefs}

The majority (88.7\%) of participants across all three groups believed that PUs are a serious or life-threatening condition. While 53\% of inpatients believed a PU will interfere a lot with their daily activities, over $85 \%$ of outpatients and peer supporters held that belief. Thirty-nine percent of participants believed that they are unlikely to develop PUs (30.3\% of outpatients, $37.5 \%$ of peer supporters and $40 \%$ of inpatients). Participants across all three groups agreed that PUs in stages three and four were more difficult to treat than in stages one and two.

$60.6 \%$ of participants believed they were able to prevent the occurrence of PUs. Participants believed that daily skin checks (80.3\%), weight shifting (86\%) and limiting sitting

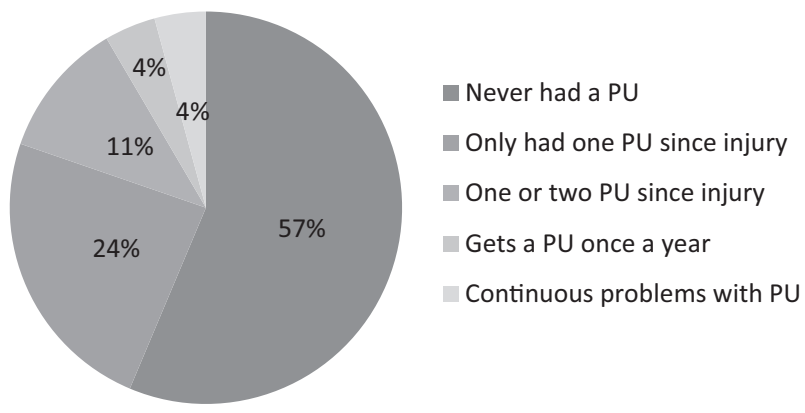

Fig. 1 Pressure-ulcer history of participants $(n=71)$ time $(80.3 \%)$ could help to prevent PUs. Most inpatients $(80 \%)$ and outpatients $(76 \%)$ and all of the peer supporters believed daily skin checks could make a difference. Of the inpatients $80 \%$ said weight shifts made a difference, $88 \%$ of the outpatients and all the peer supporters agreed. The majority $(65 \%$; 46) believed that it was not difficult to follow good skin care practices. Of the inpatients, $60 \%$ reported that skin care was not difficult. Seventy-three percent of outpatients and 50\% of the peer supporters reported the same. Those who believed that it was difficult to follow good skin care practices listed the main reasons as it taking too much time, not having the correct equipment, forgetting to do them, having too many other problems and not having anyone to help them.

\section{Practices}

The practice that showed the lowest percentage of adherence overall was performing pressure relief (49\%). Inpatients (50\%) and outpatients (39\%) scored very low in this regard, with peer supports reporting $88 \%$ compliance (Fig. 2).

\section{Skills}

Participants could perform weight shift (pressure relief) and transfers. However, weight shift was not maintained for long enough by $38 \%$ of participants. Shear forces occurred during transfers of $38 \%$ of participants.

Comparative analysis showed that an increase in time since injury had a significant positive impact on the belief that PUs would interfere with ADL $(p=0.024)$, but not on PU knowledge (0.197), nor on beliefs about the seriousness of PUs (0.722). Higher knowledge scores impacted significantly on adherence to pressure-relief practices $(p=$ 0.001). Higher levels of education did not have a significant correlation with PU history (0.205) or knowledge (0.272).

\section{Discussion}

From study findings and previous studies, it seems as if the demographic variables of being male [13, 22], having lower
Table 3 Participant's medical information in percentages (numbers in brackets are actual number of participants)

\begin{tabular}{llllr}
\hline & Total (71) & Inpatients (30) & Outpatients (33) & Peer supporters (8) \\
\hline Traumatic injuries & $89 \%(63)$ & $87 \%(26)$ & $88 \%(29)$ & $100 \%(8)$ \\
Non-traumatic injuries & $11 \%(8)$ & $13 \%(4)$ & $12 \%(4)$ & $0 \%(0)$ \\
Paraplegia & $76 \%(54)$ & $70 \%(21)$ & $85 \%(28)$ & $62 \%(5)$ \\
Tetraplegia & $24 \%(17)$ & $30 \%(9)$ & $15 \%(5)$ & $38 \%(3)$ \\
$\begin{array}{l}\text { Participants with previous } \\
\text { pressure ulcers }\end{array}$ & $43 \%(31)$ & $30 \%(9)$ & $55 \%(18)$ & $50 \%(4)$ \\
\hline
\end{tabular}


Table 4 Descriptive statistics of participants' knowledge scores

$N$ Mean \% scored (mean score SD Min Max out of 56)

\begin{tabular}{lllllll}
\hline Overall & 71 & $42.7 \%(23.9)$ & 7.8 & 17.9 & 80.4 \\
Inpatients & 30 & $40.4 \%(22.6)$ & 10.6 & 17.9 & 57.1 \\
Outpatients & 33 & $39.4 \%(22.1)$ & 12 & 19.6 & 64.3 \\
Peer supporters & 8 & $65.2 \%(36.5)$ & 12.9 & 44.6 & 80.4 \\
\hline
\end{tabular}

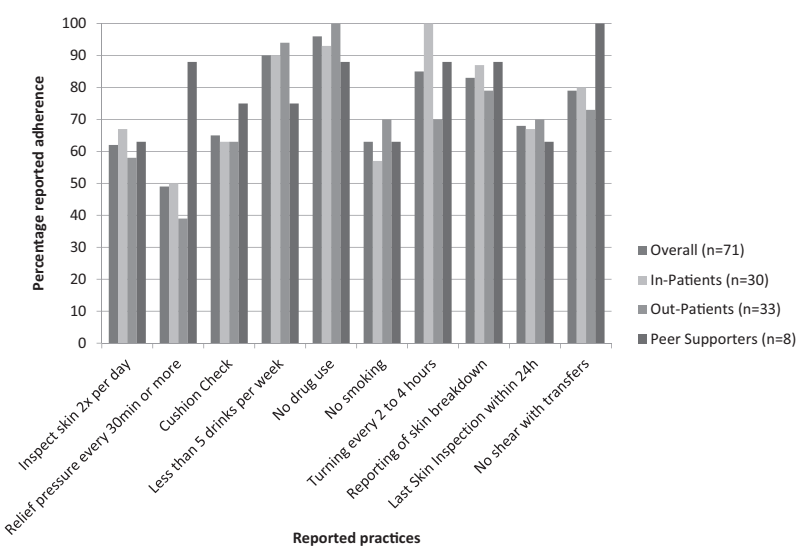

Fig. 2 Reported adherence with recommended skin care guidelines

levels of education [1, 2, 13, 22] and unemployment resulting in low income levels $[1,13,23]$ might increase the risk for PUs. One may, thus, cautiously postulate that the average client at the study centre is already at high risk of developing a PU.

The percentage of participants (43\%) who had developed a PU at some stage since injury is lower than the life-time incidence risk of $85 \%$ indicated by Zakrasek et al. [1]. As expected, however, it was higher than the prevalence rate of $29.8 \%$ found by Joseph and Wikmar [2] in a hospital-based study in the same setting as the current study. These results seems high for a group of participants of whom about $50 \%$ were less than 2 years post injury, and of whom almost half have just completed a rehabilitation program. Knowledge scores showed that $38 \%$ of the participants did not know that a red area or skin discolouration constitute a grade $1 \mathrm{PU}$ and only $11.3 \%$ of participants could describe the stages of a PU. The figure of $43 \%$ participants with previous PUs may thus reflect underreporting.

The high number of participants developing PUs in hospital is worrying. In South Africa persons who suffer a SCI are first admitted to a district or regional hospital close to where the injury has occurred. From there they are transferred to a tertiary hospital and are only referred for rehabilitation once they are medically stable. Frielingsdorf and Dunn [24] found in a study done in Cape Town that $11 \%$ of participants arrived at the tertiary hospital with PUs and a further $11 \%$ developed PUs at the tertiary hospital. The reasons for the clients arriving with PUs included delayed transfers from secondary and primary healthcare services and the use of hard fracture-boards by paramedical transport services [24].

Current study findings showed that higher knowledge scores correlated positively with better adherence to pressure relief practices. Thus a lack of knowledge might have hampered participants in practicing PU prevention strategies, even though they wanted to prevent them and regarded them as serious. Guihan et al. [16], who assessed knowledge with a similar tool, considered the $73.4 \%$ mean knowledge score, which they had found too low to effectively prevent PU. Only four of the participants in the current study scored above $73 \%$.

PU knowledge decreases over time [25]. A higher mean knowledge score was thus expected amongst inpatients than outpatients since the inpatients had just completed their rehabilitation programme. This was however, not the case. A possible explanation could be the trend to have shorter lengths of stay due to budgetary constraints. SCI is a traumatic, life changing experience and with limited inpatient stays, clients might struggle to fully absorb and understand all the information given to them [10]. Irrespective of the reasons, results showed that the inpatients did not have sufficient knowledge to prevent PUs after discharge. There are a lack of community and home-based follow-up services available to persons with disabilities in this setting [26], making this especially concerning.

One resource that is available to assist with community integration is peer supporters. The peer supporters underwent a specific training programme that included skin care and the prevention of PUs, which might have facilitated their higher mean knowledge score. Yet their scores were still lower than $73.4 \%$. This could impact on their not being able to effectively prevent PU themselves and also on teaching others to do so.

In accordance with other studies $[15,16]$ the majority of participants in the current study believed PUs to be either very serious or life threatening. The proportion of inpatients believing PUs to be life threatening compared with very serious was much lower than the same proportion of outpatients and peer supporters. Even though there was no statistically significant association between time since injury and the perceived seriousness of a PU, it would seem that people who had lived longer with a SCI and might have witnessed the possible devastating effects of PUs may view PUs in a more serious light.

Participants lacked knowledge on the importance of good general health in the fight against PUs. Factors like poor nutrition, smoking and substance abuse $[8,27,28]$ increase the risk for PUs. Smoking also impairs wound healing and, thus, the healing of existing Pus [28]. Skin checks form an 
important part of the PU prevention plan [11]. While $80.3 \%$ believed it could make a difference, only around 60\% actually performed skin checks twice a day. An important aspect of skin inspection is knowing how to do it correctly, when to do it and where the skin should be inspected, i.e. which areas are most vulnerable to PU development. Current participants most frequently name the ischia as potential sites for PU (85\%), but only $31 \%$ knew that the sacrum [11] is a high-risk area.

\section{Conclusion}

Knowledge about PUs and PU prevention were poor amongst all three the sub-groups of study participants. Although participants generally believed PUs to be serious and potentially preventable, a lack of knowledge might have hampered them in performing PU-prevention practices adequately. It also seems as if the participants did not understand the link between general health and well-being and skin health, and the subsequent prevention of PUs.

\section{Recommendations}

The findings suggest that the SCI rehabilitation programme at the Centre might benefit from implementing a structured PU-prevention programme. It is further recommended that the peer supporters undergo regular refresher courses. Use of technology, like mobile phones, to help with timing issues to ensure proper and effective weight shifts must be explored.

There is a need for large-scale epidemiological studies on SCI and PU in South Africa. The development and piloting of a comprehensive training programme regarding PUs and PU prevention through action research is recommended. Adapting the current tool to be used as a way of assessing PU risk and developing a cut-off score or score range that may be used to identify clients at high risk of developing PUs is also recommended.

Acknowledgements This manuscript is based on a research assignment for a master's degree submitted to Stellenbosch University in 2015 [29].

\section{Compliance with ethical standards}

Conflict of interest The researcher and research assistant were employed at the study centre. This could have impacted data collection and participants' answers. To try and eliminate this, the questionnaire was very structured with specific prompts that were used in the same way, for all clients. A thorough explanation of the purpose of the study was given to ensure that participants understood the importance of answering the questions truthfully.

Statement of ethics The study was approved and registered with the University of Stellenbosch, Human Research Ethics Committee (S14/
10/213). Permission to perform the study was also received from the relevant authorities. Participation in the study was voluntary and written informed consent was obtained before data collection commenced. All data are treated confidential.

Publisher's note Springer Nature remains neutral with regard to jurisdictional claims in published maps and institutional affiliations.

\section{References}

1. Zakrasek EC, Creasey G, Crew JD. Pressure ulcers in people with spinal cord injury in developing nations. Spinal Cord. 2015;53:7-13.

2. Joseph C, Wikmar LN. Prevalence of secondary medical complications and risk factors for pressure ulcers after traumatic spinal cord injury during acute care in South Africa. Spinal Cord. 2015. https://doi.org/10.1038/sc.2015.189.

3. Hagen EM. 2015 Acute complications of spinal cord injuries. World J Orthop. 2015;6:17-23.

4. Piatt JA, Nagata S, Zahl M, Li J, Rosenbluth JP. Problematic secondary health conditions among adults with spinal cord injury and its impact on social participation and daily life. J Spinal Cord Med. 2016;39:693-8.

5. Middleton JW, Lim K, Taylor L, Soden R, Rutkowski S. Patterns of morbidity and rehospitalisation following spinal cord injury. Spinal Cord. 2004;42:359-67.

6. Chan BC, Nanwa N, Mittmann N, Bryant D, Coyte PC, Houghton PE. The average cost of pressure ulcer management in a community dwelling spinal cord injury population. Int Wound $\mathrm{J}$. 2012;10:431-40.

7. Manorama A, Meyer R, Wiseman R, Bush TR. Quantifying the effects of external shear loads on arterial and venous blood flow: implications for pressure ulcer development. Clin Biomech. 2013;28:574-8.

8. Coleman S, Gorecki C, Nelson EA, Closs SJ, Defloor T, Halfens $\mathrm{R}$, et al. Patient risk factors for pressure ulcer development: systematic review. Int J Nurs Stud. 2013;50:974-1003.

9. Kruger EA, Pires M, Ngann Y, Sterling M, Rubayi S. Comprehensive management of pressure ulcers in spinal cord injury: current concepts and future trends. J Spinal Cord Med. 2013;36:572-85.

10. Hsieh, J, McIntyre, A, Wolfe, D, Lala, D, Titus, L, Campbell, K, Teasell, R. 2014. Pressure Ulcers Following Spinal Cord Injury. In Eng JJ, Teasell RW, Miller WC, Wolfe DL, Townson AF, Hsieh JTC, Connolly SJ, Noonan VK, Loh E, McIntyre A, editors. Spinal Cord Injury Rehabilitation Evidence. Version 5.0. 1-90. [Internet]. 2014 [cited 9 August 2015]. Available from: https://www.scireproject.com/wp-content/uploads/pressure_ ulcers.pdf.

11. Garber SL, Bryce TN, Gregorio-Torres TL, Ho C, Rader C. for the Consortium for Spinal Cord Medicine. Pressure-ulcer prevention and treatment following spinal cord injury: a clinical practice guideline for health-care professionals. Washington, DC: Paralyzed Veterans of America; 2014. www.mascip.co.uk/wp-content/ uploads/2015/05/CPG_Pressure-Ulcer.pdf.

12. Stansby G, Avital L, Jones K, Marsden G. Prevention and management of pressure ulcers in primary and secondary care: summary of NICE guidance. Brit Med J. 2014;348:1-5.

13. Houghton PE, Campbell KE, CPG Panel. Canadian best practice guidelines for the prevention and management of pressure ulcers in people with spinal cord injury. A resource handbook for clinicians. (Katika Integrated Communications Inc., Mississauga ON Canada, 2013). Available from: http://www.onf.org. 
14. Burkell JA, Wolfe DL, Potter PJ, Jutai JW. Information needs and information sources of individuals living with spinal cord injury. Health Info Libr J. 2006;23:257-65.

15. King RB, Porter SL, Vertiz KB. Preventive skin care beliefs of people with spinal cord injury. Rehabil Nurs. 2008;33:154-62.

16. Guihan M, Bombardier $\mathrm{CH}$. Potentially modifiable risk factors among veterans with spinal cord injury hospitalized for severe pressure ulcers: a descriptive study. J Spinal Cord Med. 2012;35:240-50.

17. Taule T, Bergfjord K, Holsvik EE, Lunde T, Stokke BH, Storlid $\mathrm{H}$, et al. Factors influencing optimal seating pressure after spinal cord injury. Spinal Cord. 2013;51:273-7.

18. Chiodo AE, Scelza WM, Kirshblum SC, Wuermser LA, Ho CH, Priebe MM. Spinal cord injury medicine. Long-term medical issues and health maintenance. Arch Phys Med Rehabil. 2007;88 (3 Suppl 1):S76-83.

19. Guihan M, Bombardier CH, Ehde DM, Rapacki LM, Rogers TJ, Bates-Jensen B, et al. Comparing multicomponent interventions to improve skin care behaviours and prevent recurrence in veterans hospitalized for severe pressure ulcers. Arch Phys Med Rehabil. 2014;95:1246-53.

20. Mokkink LB, Terwee CB, Patrick DL, Alonso J, Stratford PW, Knol DL, et al. International consensus on taxonomy, terminology, and definitions of measurement properties for health-related patient-reported outcomes: results of the COSMIN study. J Clin Epidemiol. 2010;63:737-45.

21. O'Leary Z. The essential guide to doing your research project. 3rd ed. Los Angeles: Sage; 2017.
22. Eslami V, Saadat S, Habibi Arejan R, Vaccaro AR, Ghodsi SM, Rahimi-Movaghar V. Factors associated with the development of pressure ulcers after spinal cord injury. Spinal Cord. 2012;50:899-903.

23. Saunders LL, Krause JS, Acuna J. Association of race, socioeconomic status, and health care access with pressure ulcers after spinal cord injury. Arch Phys Med Rehabil. 2012;93:972-7.

24. Frielingsdorf K, Dunn RN. Cervical spine injury outcome - a review of 101 cases treated in a tertiary referral unit. S Afr Med J. 2007;97:203-7.

25. Thietje R, Giese R, Pouw M, Kaphengst C, Hosman A, Kienast B, et al. How does knowledge about spinal cord injury-related complications develop in subjects with spinal cord injury? A descriptive analysis in 214 patients. Spinal Cord. 2011;49:43-8.

26. Hassan SAM, Visagie $S, M j i$ G. Community support services after stroke. Soc Work Pract-Res. 2011;23:1-14.

27. Saunders LL, Krause JS, Carpenter MJ, Saladin M. Risk behaviours related to cigarette smoking among persons with spinal cord injury. Nicotine Tob Res. 2014;16:224-30.

28. Gould LJ, Olney CM, Nichols JS, Block AR, Simon RM, Guihan M. Spinal cord injury survey to determine pressure ulcer vulnerability in the outpatient population. Med Hypotheses. 2014;83:552-8.

29. Visser AM, Visagie S. Do clients with spinal cord injuries know what they need to know about pressure ulcers? - A descriptive study on the knowledge, beliefs and practices about pressure ulcers. University of Stellenbosch, Stellenbosch, South Africa. 2016. 